12

\title{
Резонансы когерентного пленения населенностей в стеклянных ячейках с парами атомов цезия, изготовленных методом индукционной сварки
}

\author{
(C) В.И. Вишняков, А.О. Макаров, С.М. Игнатович \\ Институт лазерной фризики Сибирского отделения РАН, \\ 630090 Новосибирск, Россия \\ e-mail: vishnyakov@laser.nsc.ru
}

Поступила в редакцию 21.05.2021 г.

В окончательной редакции 03.06.2021 г.

Принята к публикации 07.06.2021 г.

\begin{abstract}
Продемонстрирована возможность использования индукционной сварки для изготовления спектроскопических ячеек. Данный подход позволяет улучшить оптическое качество окон, увеличить повторяемость изготовления образцов и упростить создание спектроскопических ячеек, наполненных парами щелочных металлов. Одним из возможных применений описываемой техники можно назвать бурно развивающееся направление квантовых стандартов частоты на основе когерентного пленения населенностей (КПН). Представлено описание конструкции ячеек с плоскими окнами, результаты исследований спектроскопических свойств полученных ячеек и результаты работы с полученными ячейками, используемыми в составе стандарта частоты.
\end{abstract}

Ключевые слова: VCSEL, спектроскопические ячейки, индукционная сварка, атомный стандарт частоты, КПН, буферный газ.

DOI: $10.21883 /$ OS.2021.12.51748.2320-21

\section{1. Введение}

Начиная с 60-х годов прошлого века, частоты квантово-механических переходов в атомах и молекулах активно используются в качестве частотных реперов при создании высокостабильных стандартов частоты (атомных часов) [1-3]. Переходы в атомах менее чувствительны к внешним возмущениям, таким как изменение температуры, давления, старения, и отличаются меньшим дрейфом частоты в отличие, например, от кварцевого генератора. Одним из перспективных направлений развития атомных часов можно назвать часы на основе когерентного пленения населенностей. К отличительным особенностям данного типа часов можно отнести компактность, экономичность и высокую стабильность. Уже достаточно долгое время к атомным часам проявляется интерес со стороны телекоммуникационных компаний, которые применяют стандарты частоты для синхронизации сетей [4]. Высокоточные источники сигналов крайне необходимы в системах глобального позиционирования (ГЛОНАCC, GPS, BeiDou) [5], включая случаи геопозиционирования, когда сигналы со спутников недоступны или зашумлены. Атомные стандарты частоты также используются для обмена криптографическими ключами во время защищенных сеансов связи. Однако как гражданские, так и военные потребители нуждаются в компактных приборах с минимально возможным энергопотреблением [6,7]. Один из возможных способов уменьшить габаритные размеры стандарта частоты и его энергопотребление - уменьшить физическую часть стандарта, объем которой в значительной степени зависит от размера спектроскопической ячейки. К настоящему моменту было проведено большое количество работ в сфере миниатюризации стандартов частоты: миниатюризация электронных компонентов, в том числе переход с аналоговой электроники на цифровую, переход на использование лазерного излучения взамен газоразрядной лампы, но одна из самых сложных задач создание миниатюрных спектроскопических ячеек - до сих пор привлекает внимание научного сообщества. Эту же проблему решают научные коллективы, работающие над активно развивающимся направлением квантовых магнитометров [8-11].

В составе атомных часов спектроскопическая ячейка представляет собой газонаполненную стеклянную кювету, содержащую щелочной металл. На данный момент существует несколько способов изготовления и наполнения ячеек. Изготовлению ячеек посвящено множество работ [12-14]. Отдельно хочется отметить работы по созданию спектроскопических ячеек с применением плоских окон из кристаллического граната [15] и кристаллического сапфира [16] длиною порядка $1 \mu \mathrm{m}$. Использование граната и сапфира в этих работах оправдывается желанием уменьшить взаимодействие щелочного металла с окнами ячеек.

Конструкции ячеек можно разделить на изготавливаемые человеком и сформированные при помощи автоматизированных технологических средств. Наиболее распространен способ изготовления ячеек выдувным способом (рис. 1), при котором оператор-стеклодув 


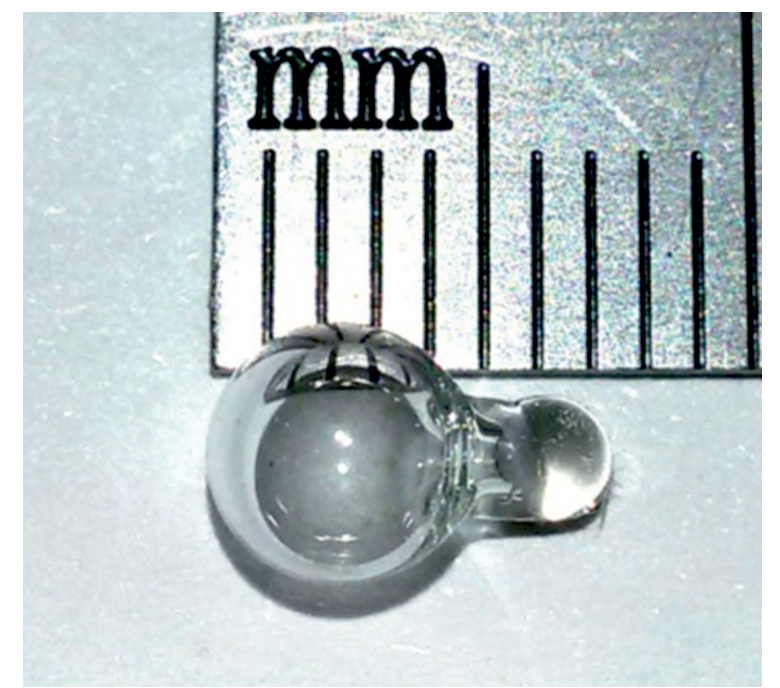

Pис. 1. Спектроскопическая ячейка, выполненная стеклодувным способом.

формирует форму ячейки и в дальнейшем припаивает ячейку к вакуумному посту, откачивает, обезгаживает, а затем напускает необходимую смесь буферных газов и щелочной металл. Хронологически спектроскопические ячейки сначала изготавливались при помощи классических стеклодувных методов. Опытный стеклодув способен выдуть миниатюрную ячейку размерами вплоть до 3-5 mm [17,18]. К недостаткам данных форм ячеек относят крайне малую повторяемость при изготовлении, ухудшение параметров пучка лазера при прохождении оптического излучения, а также увеличение конечного размера ячейки из-за отростка трубки, через которую ячейка наполнялась. Стоит отметить тот факт, что для выполнения данных работ должен быть привлечен специалист-стеклодув достаточно высокой компетенции и опыта. Преимуществом данного метода можно назвать относительную простоту изготовления как самих ячеек, так и способа их дальнейшего наполнения.

Стоит отметить прогресс в создании другого типа ячеек, основанных на технологии микроэлектромеханических систем (МЭМС - microelectromechanical systems (MEMS)) [19]. При помощи отработанных технологических процессов типичный размер получаемого образца уменьшился до порядка $1 \mathrm{~mm}^{3}$ [20]. Отметим еще одну работу, в которой научный коллектив проводил работы по уменьшению температуры, необходимой для приваривания окон к основанию ячейки, до $<140^{\circ} \mathrm{C}[21]$. Спектроскопические ячейки, изготовленные по технологии МЭМС, отличаются хорошим оптическим качеством входных/выходных окон. Однако у данной технологии существует и ряд недостатков.

- Достаточно высокотехнологичный процесс, требующий наличия дорогостоящего оборудования.

- Необходимость нагрева окон ячеек: металл конденсируется на окнах, таким образом затрудняя про- хождение оптического излучения. Это происходит из-за разности в коэффициентах теплопроводности используемых материалов - для окон используют пирекс, а для подложки - кремний.

Из-за необходимости испарять сконденсированный металл с окон ячейки появляется необходимость в создании нагревателя. На данный момент научным сообществом было предложено несколько схем нагрева ячеек. Среди них можно отметить работу по напылению электрических контактов на поверхности окон [22]. Однако такая схема нагрева создает неоднородное магнитное поле, что в свою очередь уширяет резонанс КПН, из-за чего такие ячейки становятся менее предпочтительными для экспериментов со стандартами частоты. Недавно был предложен еще один вариант нагрева ячейки без создания паразитного магнитного поля: нагрев ячейки при помощи волновода от инфракрасного лазерного диода с длиной волны $808 \mathrm{~nm}$ [23].

Процесс анодной сварки связан с длительным нагревом (несколько часов при температуре порядка $300^{\circ} \mathrm{C}$ ). Данный процесс ведет к тому, что в ячейке появляются посторонние газы, ухудшающие параметры резонансов КПН.

Стоит отметить одно из перспективных направлений по изготовлению ячеек для малогабаритных стандартов частоты [24]. Авторы статьи использовали „гибридный“ способ изготовления ячеек: в процессе изготовления была использована кремниевая подложка с небольшими углублениями, сваренная со стеклом при помощи анодной сварки. Затем полученный „пирог“ помещали в печь при температуре $850^{\circ} \mathrm{C}$, при этом стеклянная поверхность плавилась и под давлением оставшегося внутри газа формировалась почти идеальная полусфера. Затем нижняя часть кремниевой подложки протравливалась и при помощи пипетки внутренность наполнялась щелочным металлом. Преимуществом данного метода можно назвать возможность массового изготовления ячеек и их миниатюрный размер (диаметр сферы $\sim 1 \mathrm{~mm}$ ). К недостаткам можно отнести достаточно сложный и высокотехнологичный процесс изготовления и наполнения.

Качество оптической поверхности окон спектроскопических ячеек крайне важно при проведении экспериментов, в том числе для создания квантового стандарта частоты. Среди множества возможных вариантов изготовления ячеек в литературе до сих пор не встречался метод изготовления при помощи индукционной сварки. В нашей работе индукционная сварка используется для сваривания плоских окон и цилиндрического тела ячейки. Разными авторами было выдвинуто множество вариантов изготовления, наполнения и тестирования полученных ячеек, но до сих пор не было найдено единого варианта изготовления ячеек, отвечающего всем требованиям качества изготовления оптических поверхностей, стоимости, сложности изготовления и др.

Для серийного изготовления атомных стандартов частоты необходимо налаженное производство ячеек, наполненных смесью буферных газов и паров изотопов 
щелочных металлов. Такие ячейки могут использоваться в качестве основы микроволнового стандарта частоты (атомных часов). Безбуферные варианты этих ячеек также могут быть использованы в качестве репера при калибровке источников лазерного излучения по длине волны.

Под действием многочастотного когерентного излучения в многоуровневой квантовой системе возникает состояние суперпозиции, в результате которого квантовая система перестает взаимодействовать с излучением. Данный эффект сильно зависит от отстройки разности частот спектральных компонент лазерного поля от частоты сверхтонкого расщепления основного состояния в атоме. Поэтому при изменении разности частот бихроматического поля в проходящем излучении наблюдается узкий пик прозрачности среды, называемый резонансом КПН. КПН был впервые изучен группой ученых в 1976 г. при наблюдении флуоресценции в ячейках, наполненных парами натрия [25].

\section{2. Исследование спектроскопических ячеек}

\section{1. Изготовление ячеек}

В нашей работе использовались спектроскопические ячейки, изготовленные на заводе АО „Экран-Оптические системы“, расположенном в г. Новосибирск, Российская Федерация. Конструктивно ячейки АО „ЭкранОптические системы“ состоят из двух плоских окон, стеклянного цилиндра и магнитопроводящих колец, необходимых в процессе изготовления ячейки. Особенностью данных ячеек можно назвать способ скрепления окон и цилиндрического основания ячейки. Окна герметично привариваются к цилиндрическому основанию при помощи индукционной сварки. При этом магнитопроводящее кольцо „погружено“ в расплавленное стекло со всех сторон, ввиду чего щелочной металл, находящийся внутри ячейки, не входит в контакт с кольцом.

Данный метод изготовления позволяет значительно уменьшить оптические искажения в окнах ячеек, которые происходят при производстве другими способами, например стеклодувным способом. Так как процесс индукционной сварки продолжается всего несколько секунд, плоские окна не подвергаются значительному разогреву и не деформируются. Следовательно, проходящее через ячейку оптическое излучение не преломляется и не рассеивается, как в случае с маленькими сферическими ячейками. На рис. 2 сверху показана пустая ячейка - заготовка. Так как в процессе производства ячеек участвует магнитопроводящий материал, было необходимо оценить вносимые неоднородности в магнитное поле. Для учета вносимового искажения магнитного поля от этих колец был выполнен теоретический расчет при помощи метода конечных элементов (МКЭ, англ. FEM - finite element method). Начальным условием
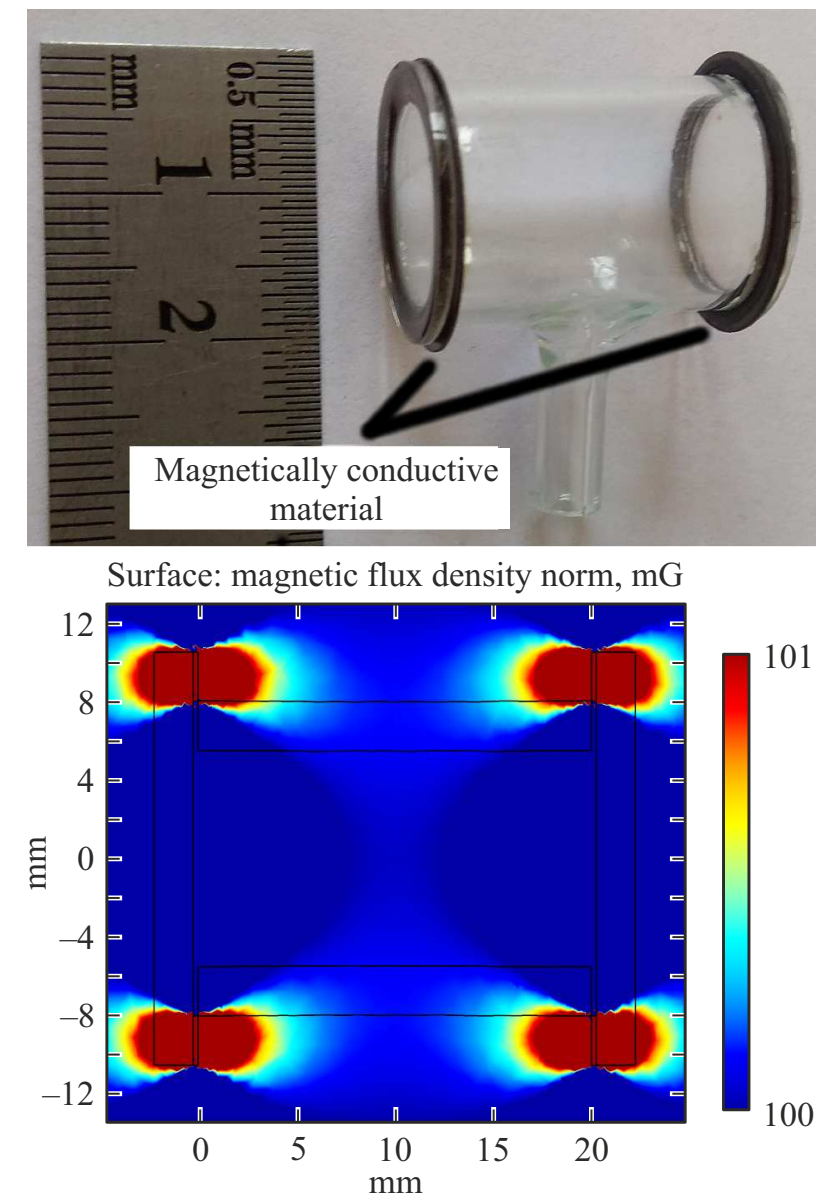

Рис. 2. Фотография ячейки (сверху), результат МКЭ исследования (внизу).

модели устанавливалась индукция внешнего магнитного поля, равная $100 \mathrm{mG}\left(10^{-5} \mathrm{~T}\right)$, что является типичной величиной, необходимой для расщепления зеемановских подуровней в атомах цезия. Согласно результату расчета (рис. 2, внизу), кольца вносят искажения магнитного поля на уровне $40 \mu \mathrm{G}\left(4 \cdot 10^{-9} \mathrm{~T}\right)$ в центре ячейки.

\section{2. Наполнение ячеек}

Для наполнения ячеек щелочным металлом и буферным газом использовался вакуумный пост. Вакуумный пост состоял из форвакуумного, турбомолекулярного насосов, металлической и стеклянной арматуры. Форвакуумный и турбомолекулярный насосы вместе позволяют создать разряжение порядка $10^{-5}$ Torr. Процесс наполнения ячеек можно условно разделить на три этапа.

1) Подпаивание ячеек к вакуумному посту. Предварительное обезгаживание.

2) Наполнение ячеек щелочным металлом и смесью буферных газов.

3) Отпаивание ячеек от поста. 
Остановимся на каждом пункте подробнее. Вначале оператор подпаивает ячейки с плоскими окнами и контейнер с изотопически чистым цезием к стеклянной гребенке, которая в свою очередь подпаяна к вакуумному посту. Затем из поста откачивается оставшаяся внутри атмосфера. Остаточное давление при этом составляет порядка $10^{-5}$ Torr. Затем весь объем заполняется инертным газом - аргоном. Контейнер с щелочным металлом надламывается и металл перемещается в стеклянную гребенку с подпаянными ячейками. В дальнейшем гребенка подогревается и подогретый цезий переносится в исследуемые в данной работе ячейки. Затем инертный газ откачивается и емкость заполняется смесью буферных газов необходимого давления. После этого стеклодув отпаивает ячейки от гребенки. Так как габаритные размеры ячеек являются ключевым фактором, влияющим на компактность физического блока стандарта частоты, то уменьшив отросток ячейки, нашей команде удалось уменьшить и габаритные размеры ячейки.

\section{3. Экспериментальная установка}

В нашей установке ячейка была смонтирована в термокожух, выполненный из немагнитного материала латуни. Для обеспечения однородности нагрева ячейки на термокожухе было необходимо установить несколько нагревательных элементов. Использование двух нагревателей, смонтированных симметрично на торцах термокожуха, улучшило распределение тепла, таким образом уменьшив температурный градиент по ячейке.

Для отработки температурных возмущений на торцах термокожуха были смонтированы термодатчики - терморезисторы с отрицательным температурным коэффициентом сопротивления (Negative temperature coefficient (NTC)). При помощи платы обработки данных и АЦП (аналого-цифровой преобразователь) была осуществлена термостабилизация с точностью до нескольких милликельвин. Для расщепления зеемановских подуровней и изолирования „часового“ перехода „0-0“ требовалось приложить постоянное магнитное поле. В нашей установке для создания однородного продольного магнитного поля использовался соленоид. Для экранирования от внешних магнитных возмущений был использован трехслойный цилиндрический экран из материала с высокой магнитной проницаемостью (пермаллой марки 79НМ). При помощи феррозондового датчика было измерено магнитное поле внутри пермаллоевых экранов. Величина магнитного поля внутри экранов была в 900 раз меньше, чем снаружи экранов. Соответственно магнитное поле Земли удалось экранировать до долей миллигаусс.

Для тестирования спектроскопических ячеек была собрана оптическая установка, показанная на рис. 4. В работе использовался лазер с вертикальным резонатором (ЛВР, vertical-cavity surface-emitting laser (VCSEL)), излучающий на длине волны $895 \mathrm{~nm}$ (D1 линия цезия). Измеренная спектральная ширина линии лазера $a$
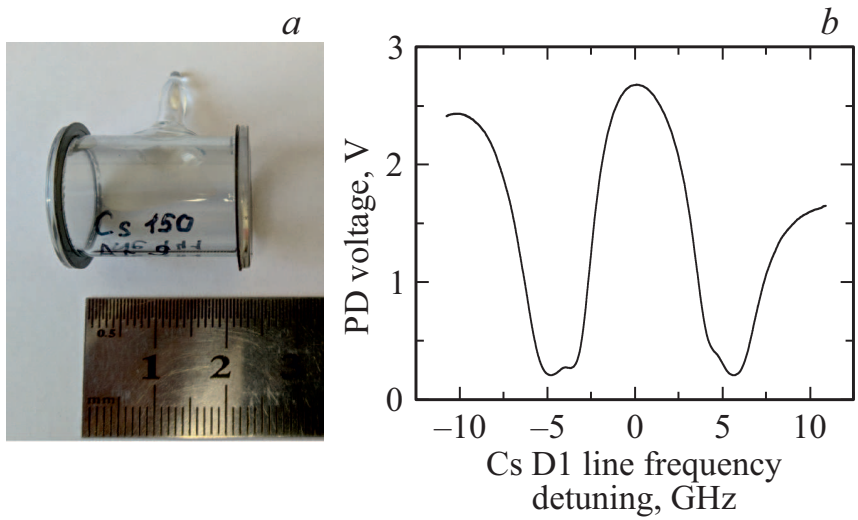

c
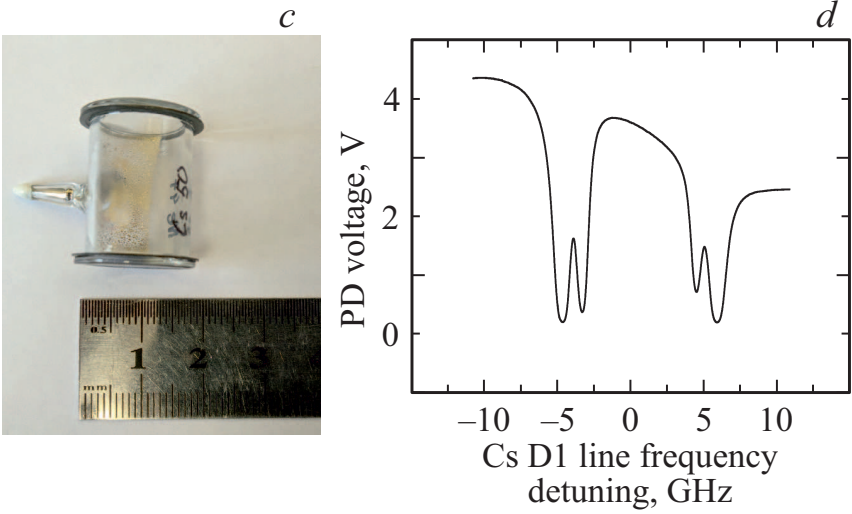

Рис. 3. На рисунках изображены график поглощения лазерного излучения $(b)$ в ячейке, наполненной 150 Torr смесью буферных газов $(a)$ при температуре $70^{\circ} \mathrm{C}$, и график поглощения лазерного излучения $(d)$ в ячейке, наполненной 50 Torr смесью буферных газов (c) при температуре $50^{\circ} \mathrm{C}$.

составила $20 \mathrm{MHz}$. При помощи ПЗС-камеры (CCD „charge-coupled device“ ${ }^{6}$ был измерен профиль пучка лазера. Диаметр пучка лазера составил около $1 \mathrm{~mm}$. Излучение лазера проходило через $\lambda / 4$ пластинку для формирования круговой поляризации. Такой вариант наблюдения КПН резонанса считается одним из самых компактных вариантов атомных часов [26]. Для аттенуирования оптической мощности был применен градиентный нейтральный фильтр. Так как в ячейках используются окна диаметром $18 \mathrm{~mm}$, то для увеличения области взаимодействия лазерного луча с щелочным металлом был использован телескоп, сформированный двумя линзами. При помощи телескопа диаметр пучка лазера был увеличен в 4 раза. После прохождения поглощающей ячейки излучение собиралось при помощи короткофокусной линзы и фотоприемника и фиксировалось на осциллографе с функцией записи сигналов.

Для получения КПН резонанса использовалась СВЧ модуляция частоты лазера на частоте $4.596 \mathrm{GHz}$. Стабилизация оптической частоты лазера осуществлялась системой автоматической подстройки частоты по линии поглощения D1 в атоме Cs. Ключевым элементом системы автоподстройки частоты является СВЧ-генератор. Сформированный генератором сигнал, в спек- 


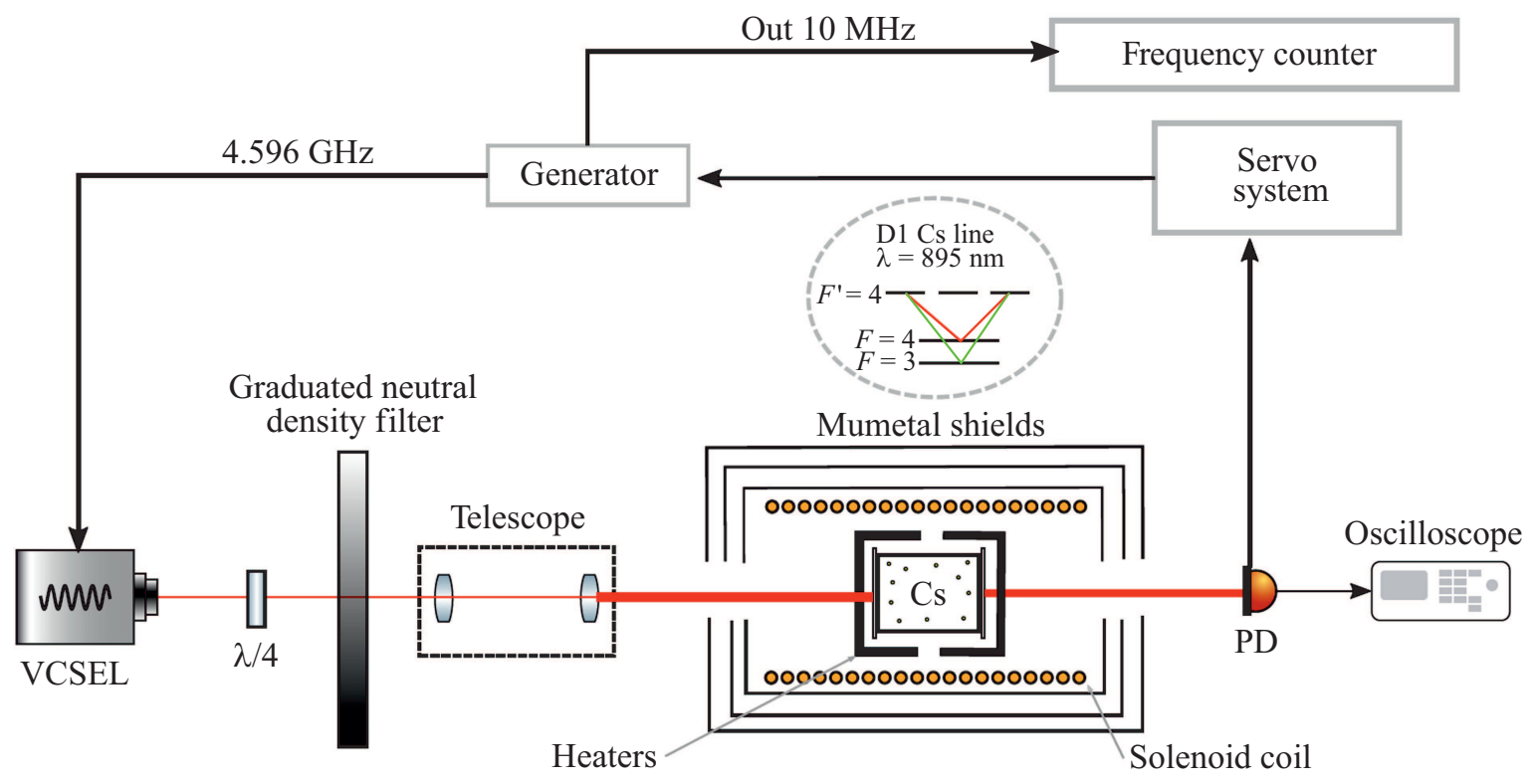

Рис. 4. Схема установки по изучению спектроскопических свойств ячеек.

тральном представлении состоит из несущей частоты и двух боковых полос („сайдбенды“ - sidebands). Разность частот, возникающая между \pm 1 сайдбендами, совпадает с частотой сверхтонкого расщепления основного состояния в атоме цезия. СВЧ-генератор, в свою очередь, „привязывается“ по частоте к кварцевому генератору, управляемому напряжением (ГУН). Модуляция излучения осуществлялась путем подмешивания СВЧ в ток накачки диодного лазера через Т-образный фильтр (Bias-Tee). Стабилизация частоты СВЧ-генератора осуществлялась системой автоматической стабилизации по резонансу КПН, имеющей схожий принцип с методом Паунда-Древера-Холла (Pound-Drever-Hall) [27]. СВЧ-генератор имеет выходной блок для формирования прямоугольного сигнала частотой $10 \mathrm{MHz}$. Сигнал $10 \mathrm{MHz}$ подавался на частотомер, где обрабатывался и записывался. В качестве опоры на частотомер подавался сигнал пассивного водородного стандарта частоты Ч1-1007 производства ЗАО „Время-Ч“.

\section{3. Результаты}

Нами были изготовлены две ячейки с плоскими окнами, наполненные щелочным металлом - цезием и смесью буферных газов. Первая ячейка была наполнена смесью с общим давлением 50 Torr $(\mathrm{Ar}-3$ Topp и $\mathrm{Ne}-$ 47 Torr). Вторая ячейка была наполнена смесью с общим давлением 150 Torr (Ar - 9 Torr и Ne - 141 Torr). Каждая ячейка была смонтирована в термостабилизируемый корпус, совмещенный с соленоидом, и помещена внутрь системы многослойных пермаллоевых экранов. На рис. 3 представлены фотографии изготовленных ячеек и профили поглощения лазерного излучения для

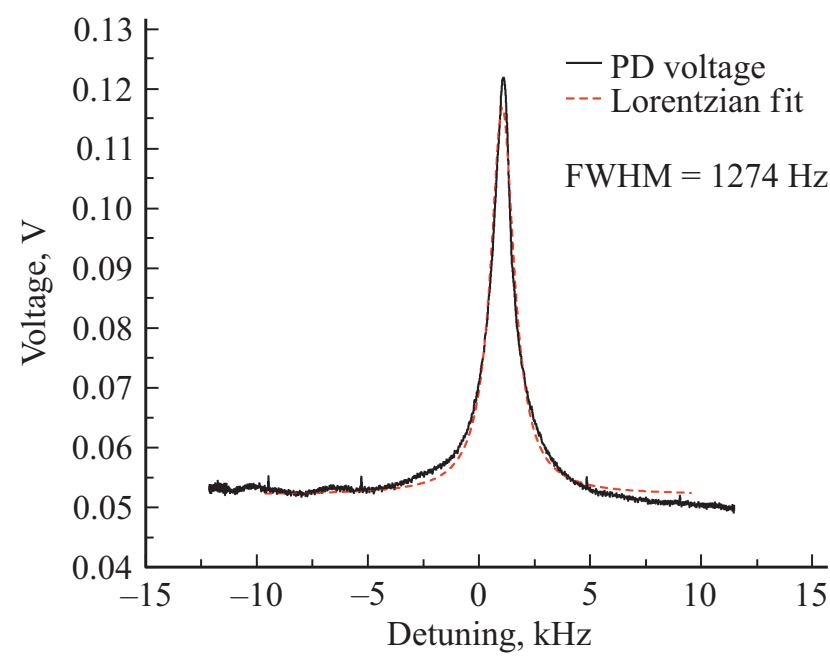

Рис. 5. КПН резонанс, наблюдаемый при температуре ячейки $36^{\circ} \mathrm{C}$ и оптической мощности $20 \mu \mathrm{W}$.

каждой ячейки. Отметим, что на рис. $3, b$ сверхтонкое расщепление возбужденного уровня практически не разрешается. Очевидно, это является следствием сильного столкновительного уширения спектральной линии в ячейке с давлением 150 Torr буферного газа. Соответствующие коэффициенты для такого уширения равны: $\mathrm{Ar}-18.3 \mathrm{MHz} /$ Torr и $\mathrm{Ne}-10.8 \mathrm{MHz} /$ Torr [28]. Из рис. 3 видно, что количество щелочного металла, оставшегося в ячейке после отпайки, достаточно для проведения спектроскопических экспериментов.

После сборки экспериментальной установки были проведены эксперименты по изучению поглощения щелочного металла в ячейках. ЛВР перестраивался по длине волны при помощи встроенного элемента Пель- 

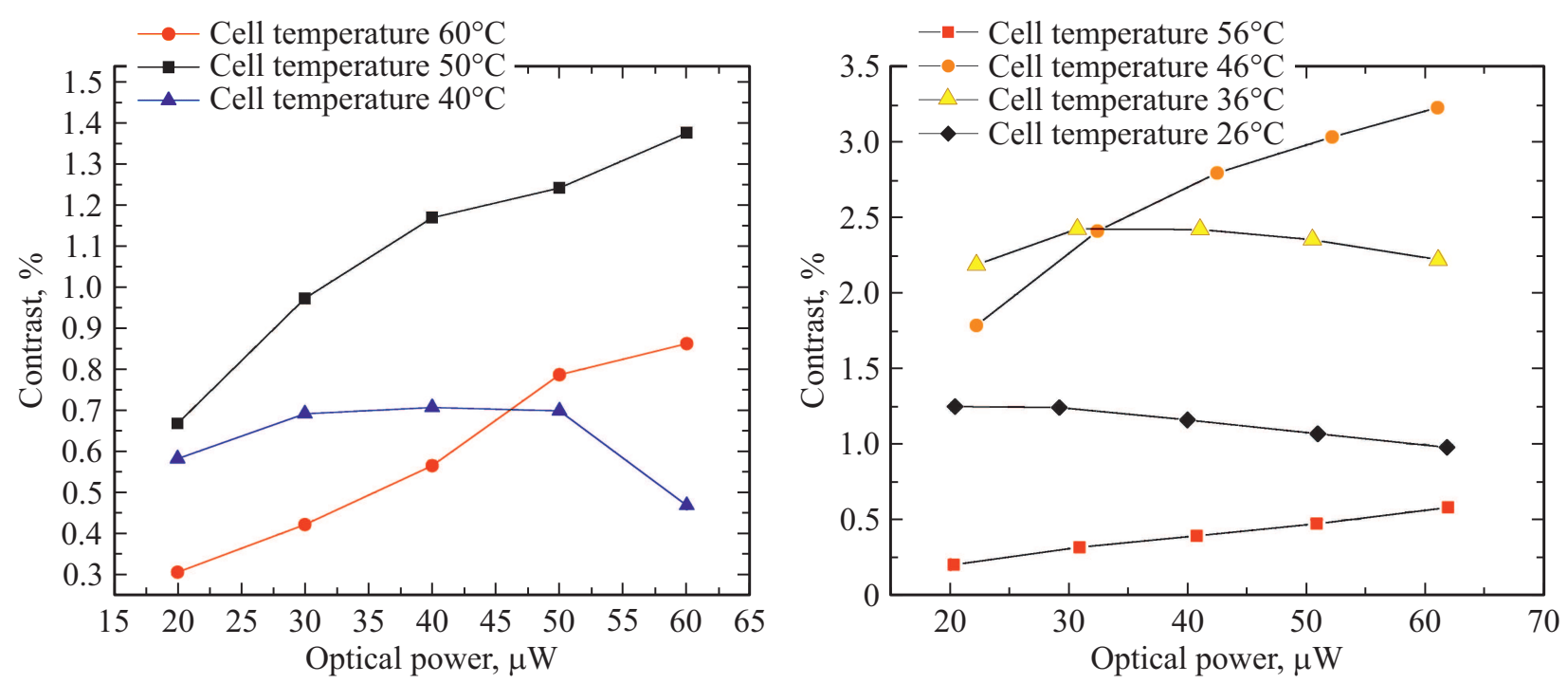

Рис. 6. Зависимость контраста от оптической мощности в ячейках с давлением буферных газов - 150 Torr (расположена слева) и 50 Torr (расположена справа).
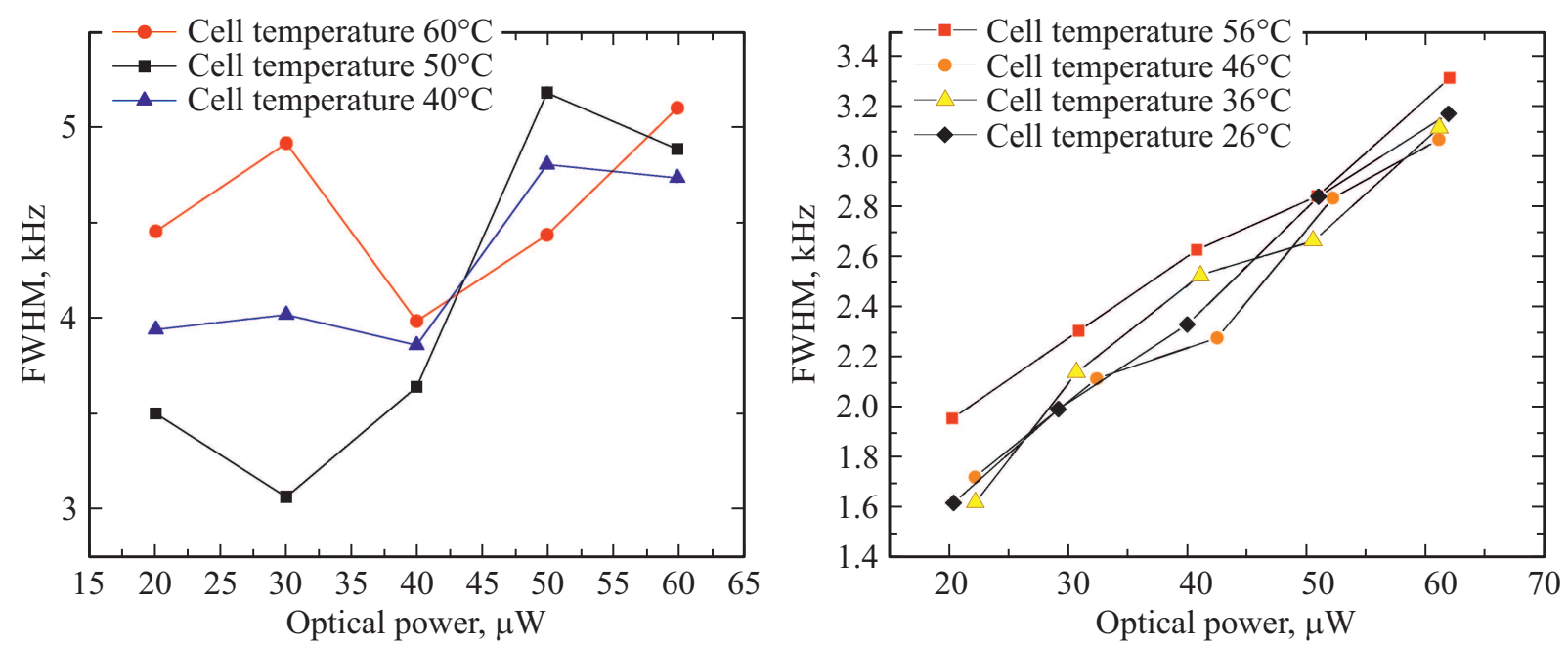

Рис. 7. Зависимость полной ширины на полувысоте КПН резонанса для ячеек: 150 Torr (расположен слева) и 50 Тоrr (расположен справа).

тье. Для получения графиков зависимости поглощения от длины волны сканировался ток лазера. Для оценки спектроскопических качеств полученных ячеек были измерены ширины КПН резонансов. На рис. 5 изображен КПН резонанс, полученный при сканировании частоты СВЧ-генератора.

Одним из важных параметров, влияющих на кратковременную стабильность стандарта частоты, является отношение амплитуды КПН резонанса к величине подложки контура поглощения (фона). Данный параметр в литературе часто называют контрастом резонанса. В ходе работы были измерены контраст и ширина КПН резонансов. Нами были проведены измерения ширины КПН резонанса и контраста относительно изменения оптической мощности, поступающей в ячейку.
Для определения оптимальных параметров для работы стандарта частоты были измерены контраст и ширина КПН резонансов для диапазона оптических мощностей. На рис. 6 изображены результаты измерений, выполненных для двух ячеек. Стоит отметить, что контраст для ячейки, наполненной 50 Torr смесью буферных газов, в два раза лучше, чем для ячейки с 150 Torr. Уменьшение контраста с ростом давления буферного газа может быть связано с тем, что при одной и той же температуре в ячейке с 150Torr давления контур поглощения имеет меньшую амплитуду, чем в ячейке с 50 Torr давления. Таким образом, в последнем случае фон у резонанса КПН меньше и, следовательно, контраст выше. Обратив внимание на графики зависимости полной ширины на полувысоте (рис. 7), можно отметить 


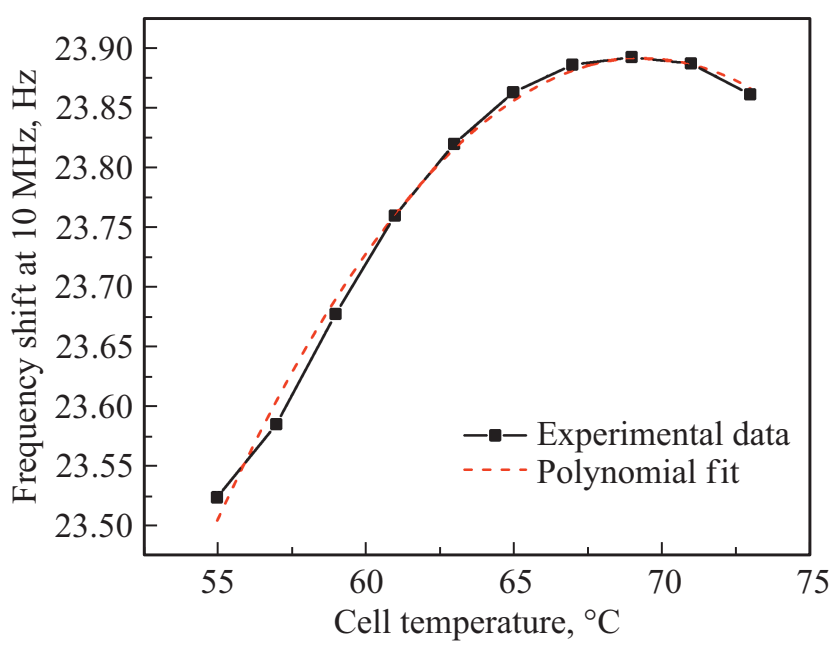

Рис. 8. Зависимость сдвига частоты $10 \mathrm{MHz}$ от изменения температуры ячейки с давлением буферных газов 150 Torr (сплошная линия); аппроксимация полиномом второго порядка (штриховая линия) [29].

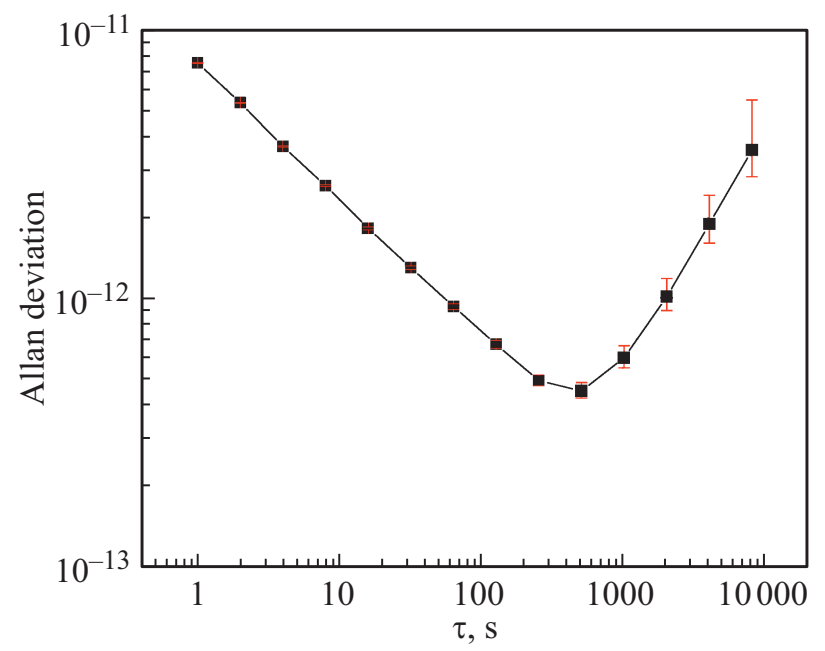

Рис. 9. Девиация Аллана для изготовленной ячейки с общим давлением 50 Torr.

более узкий резонанс для ячейки с 50 Torr буферного газа.

Для улучшения долговременной стабильности стандарта частоты было необходимо уменьшить влияние внешних условий, в том числе температурных. Для этого необходимо найти точку, наименее чувствительную к вариациям температуры. Ячейка была помещена внутрь пермаллоевых экранов, которые, в свою очередь, были закрыты крышками, уменьшающими конвективный снос тепла с нагретой ячейки. Для проведения эксперимента были задействованы кольца обратной связи и ступенчато изменялась температура ячейки. Затем из записи изменения частоты $10 \mathrm{MHz}$ был построен график 8 . Из этого графика видно, что для ячейки с 150 Torr буферного газа есть точка со слабой чувствительностью частоты стандарта к флуктуациям температуры - это экстремум приведенной зависимости. Для ячейки с 50 Torr буферных газов точку, нечувствительную к температурным изменениям в диапазоне $25^{\circ}-70^{\circ} \mathrm{C}$, найти не удалось. Возможной причиной можно назвать недостаточную точность поддержания пропорции смеси буферных газов при наполнении этой конкретной ячейки.

Для осуществления проверки ячеек на пригодность применения в качестве основы квантового стандарта частоты ячейка с 50 Torr буферного газа была смонтирована и термостабилизирована. На рис. 9 изображена девиация Аллана, характеризующая относительную нестабильность частоты для нашей экспериментальной установки, использующей изготовленную ячейку.

\section{4. Заключение}

В нашей работе были исследованы стеклянные ячейки цилиндрической формы с парами щелочного металла, окна в которых были приварены с помощью индукционной сварки. Отличительными особенностями данной технологии изготовления ячеек от традиционной стеклодувной являются более высокое качество окон (отсутствие заметных искажений светового пучка), простота изготовления и, как следствие, возможность массового производства. Для демонстрации потенциальных возможностей ячеек мы провели серию экспериментов по наблюдению резонансов (КПН) в ячейках, наполненных смесью буферных газов и парами атомов цезия. Характеристики наблюдаемых резонансов не уступают тем, что обычно наблюдаются в ячейках, изготовленных стеклодувным способом. Кроме того, был создан лабораторный макет микроволнового квантового стандарта частоты на основе резонансов КПН. Измеренная относительная стабильность частоты, характеризуемая девиацией Аллана, составила $\sigma_{\mathrm{y}} \approx 7.5 \cdot 10^{-12}$ за $1 \mathrm{~s}$ усреднения и $6 \cdot 10^{-13}$ за $1000 \mathrm{~s}$. Таким образом, была продемонстрирована довольно высокая стабильность частоты, не уступающая передовым аналогам компактных атомных часов на основе резонансов КПН [22,27]). Следовательно, предложенный метод изготовления ячеек может быть востребован в различных лазерных технологиях, в том числе для создания высокостабильных компактных атомных часов.

\section{Благодарности}

Авторы благодарны М.Н. Скворцову, И.С. Месензовой и Д.В. Бражникову за полезные дискуссии в ходе подготовки статьи.

\section{Финансирование работы}

Исследование выполнено при финансовой поддержке РФФИ и Министерства науки и инновационной политики Новосибирской области в рамках научного проекта № 19-42-543001. 


\section{Конфликт интересов}

Авторы заявляют, что у них нет конфликта интересов.

\section{Список литературы}

[1] Багаев С.Н., Чеботаев В.П. // УФН. 1986. Т. 148, № 1. C. 143-178. doi 10.3367/UFNr.0148.198601g

[2] Тайченачев А.В., Юдин В.И., Багаев С.Н. // УФН. 2016. T. 186. № 2. C. 193-205. doi 10.3367/UFNr.0186.201602j.0193

[3] Riley W.J. // IEEE UFFC-S History. 2019. http://ieee-uffc.org/about-us/history/a-history-oftherubidium-frequency-standard.pdf

[4] Kusters J.A., Adams C.A. // RF design. 1999. V. 22. P. 28-39.

[5] Vig J. // IEEE Transactions on Ultrasonics, Ferroelectrics, and Frequency Control. 1993. V. 40. N 5. P. 522-527. doi 10.1109/58.238104

[6] Serkland D.K., Geib K.M., Peake G.M., Lutwak R., Rashed A., Varghese M., Tepolt G., Prouty M. // Vertical-Cavity Surface-Emitting Lasers XI, Choquette K.D., Guenter J.K., International Society for Optics и Photonics. 2007. V. 6484. P. 48-57. doi 10.1117/12.715077

[7] Lutwak R., Emmons D., English T., Riley W., Duwel A., Varghese M., Serkland D., Peake G. 34th Annual Precise Time and Time Interval Systems Applications Meeting. 2004.

[8] Shah V., Knappe S., Schwindt P.D.D., Kitching J. // Nature Photonics. 2007. V. 1. N 11. P. 649-652. doi 10.1038/nphoton.2007.201

[9] Johnson C., Schwindt P.D. // 2010 IEEE International Frequency Control Symposium. 2010. V. 371. P.375. doi 10.1109/FREQ.2010.5556310

[10] Woetzel S., Schultze V., IJsselsteijn R., Schulz T., Anders S., Stolz R., Meyer H.-G. // Review of Scientific Instruments. 2011. V. 82. N 3. P. 033111 . doi 10.1063/1.3559304

[11] Tsujimoto K., Hirai Y., Sugano K., Tsuchiya T., Tabata O. // Electronics and Communications in Japan. 2013. V. 96. N 5. P. 58-66. doi 10.1002/ecj.10432

[12] Grzebyk T., Górecka-Drzazga A., Dziuban J.A., Dankovic T., Feinerman A., Busta H. // Procedia Engineering. 2014. V. 87. P. 891-894. doi 10.1016/j.proeng.2014.11.298

[13] Hasegawa M., Chutani R.K., Gorecki C., Boudot R., Dziuban P., Giordano V., Clatot S., Mauri L. // Sensors and Actuators A: Physical. 2011. V. 167. P. 594-601. doi 10.1016/j.sna.2011.02.039

[14] Losev S., Sevostianov D., Vassiliev V., Velishansky V. // Physics Procedia. 2015. V. 71. P. 242-246. doi 10.1016/j.phpro

[15] Саргсян А., Амирян А., Карталева С., Саркисян Д. // ЖЭТФ. 2017. C. 54-61. doi 10.7868/s0044451017070057

[16] Peyrot T., Sortais Y.R.P., Browaeys A., Sargsyan A., Sarkisyan D., Keaveney J., Hughes I.G., Adams C.S. // Phys. Rev. Lett. 2018. V. 120. P. 243-401. doi 10.1103/PhysRevLett.120.243401

[17] Balabas M.V., Budker D., Kitching J., Schwindt P.D.D., Stalnaker J.E. // J. Opt. Soc. Am. B. 2006. V. 23. N 6. P. 1001-1006. doi 10.1364/JOSAB 23.001001

[18] Игнатович С.М., Скворцов М.Н., Вишняков В.И., Бражников Д.В., Квашнин Н.Л., Васильев В.А., Юдин В.И., Тайченачев А.В., Багаев С.Н. „Атомные часы на эффекте когерентного пленения населенностей“, Материалы IX Международного симпозиума. Метрология времени и пространства, сентябрь. 2018.
[19] Kroemer E., Hafiz M.A., Maurice V., Fouilland B., Gorecki C., Boudot R. // Opt. Express. 2015. V. 23. N 14. P. 18373-18380. doi 10.1364/OE.23.018373

[20] Pétremand Y., Affolderbach C., Straessle R., Pellaton M., Briand D., Mileti G., de Rooij N.F. // J. Micromechanics and Microengineering. 2012. V. 22. N 2. P. 025013. doi 10.1088/0960-1317/22/2/025013

[21] Straessle R., Pellaton M., Affolderbach C., P?etremand Y., Briand D., Mileti G., de Rooij N.F. // J. Appl. Phys. 2013. V. 113. N 6. P. 064501 . doi 10.1063/1.4789942

[22] Knappe S., Shah V., Schwindt P.D.D., Hollberg L., Kitching J., Liew L.-A., Moreland J. // Appl. Phys. Lett. 2004. V. 85. N 9. P. 1460-1462. doi.org/10.1063/1.1787942

[23] Oelsner G., IJsselsteijn R., Scholtes T., Krüger A., Schultze V., Seyffert G., Werner G., Jäger M., Chwala A., Stolz R. Integrated optically pumped magnetometer for measurements within Earth's magnetic field, 2020. arXiv: 2008. 01570 [physics.atom-ph].

[24] Eklund E.J., Shkel A.M., Knappe S., Donley E., Kitching J. // Sensors and Actuators A: Physical. 2008. V. 143. P. 175-180. doi 10.1016/j.sna.2007.10.006

[25] Alzetta G., Gozzini A., Moi L., Orriols G. // Nuov Cim B. 1976. V. 36. P. 5-20. doi 10.1007/BF02749417

[26] Kitching J. // Appl. Phys. Rev. 2018. V. 5. N 3. P. 031302. doi 10.1063/1.5026238

[27] Skvortsov M.N., Ignatovich S.M., Vishnyakov V.I., Kvashnin N.L., Mesenzova I.S., Brazhnikov D.V., Vasil'ev V.A., Taichenachev A.V., Yudin V.I., Bagayev S.N., Blinov I.Y., Pal'chikov V.G., Samokhvalov Y.S., Parekhin D.A. // Quantum Electronics. 2020. V. 50. N 6. P. 576-580. doi 10.1070/qel17339

[28] Pitz G.A., Wertepny D.E., Perram G.P. // Phys. Rev. A. 2009. V. 80. P. 062 718. doi 10.1103/PhysRevA.80.062718

[29] Kozlova O., Guérandel S., de Clercq E. // Phys. Rev. A. 2011. V. 83. P. 062714. doi 10.1103/PhysRevA.83.062714 